\title{
Experimental Investigation of Quantum Decay at Short, Intermediate, and Long Times via Integrated Photonics
}

\author{
Andrea Crespi, ${ }^{1,2}$ Francesco V. Pepe ${ }^{3}$ Paolo Facchi, ${ }^{4,3}$ Fabio Sciarrino, ${ }^{5}$ Paolo Mataloni, ${ }^{5,2}$ \\ Hiromichi Nakazato, ${ }^{6}$ Saverio Pascazio, ${ }^{4,3,7}$ and Roberto Osellame ${ }^{2,1, *}$ \\ ${ }^{1}$ Dipartimento di Fisica, Politecnico di Milano, I-20133 Milano, Italy \\ ${ }^{2}$ Istituto di Fotonica e Nanotecnologie, Consiglio Nazionale delle Ricerche, I-20133 Milano, Italy \\ ${ }^{3}$ INFN, Sezione di Bari, I-70126 Bari, Italy \\ ${ }^{4}$ Dipartimento di Fisica and MECENAS, Università di Bari, I-70126 Bari, Italy \\ ${ }^{5}$ Dipartimento di Fisica, Sapienza Università di Roma, I-00185 Roma, Italy \\ ${ }^{6}$ Department of Physics, Waseda University, 169-8555 Tokyo, Japan \\ ${ }^{7}$ Istituto Nazionale di Ottica (INO-CNR), I-50125 Firenze, Italy
}

(Received 30 October 2018; published 3 April 2019)

\begin{abstract}
The decay of an unstable system is usually described by an exponential law. Quantum mechanics predicts strong deviations of the survival probability from the exponential: Indeed, the decay is initially quadratic, while at very large times it follows a power law, with superimposed oscillations. The latter regime is particularly elusive and difficult to observe. Here we employ arrays of single-mode optical waveguides, fabricated by femtosecond laser direct inscription, to implement quantum systems where a discrete state is coupled and can decay into a continuum. The optical modes correspond to distinct quantum states of the photon, and the temporal evolution of the quantum system is mapped into the spatial propagation coordinate. By injecting coherent light states in the fabricated photonic structures and by measuring a small scattered fraction of such light with an unprecedented dynamic range, we are able to experimentally observe not only the exponential decay regime, but also the quadratic Zeno region and the power-law decay at long evolution times.
\end{abstract}

DOI: 10.1103/PhysRevLett.122.130401

The exponential decay law is commonly associated with the probability that a system, initially prepared in an unstable state (such as an excited atomic level or an unstable elementary particle), is observed in the same state after some time [1,2]. Actually, for quantum mechanical unstable states, decay can be only approximately exponential [3-5]: At short times the survival probability is quadratic, while at long times it is dominated by a power law [see Fig. 1(a)]. The aforementioned features of quantum evolution are consequences of first principles and represent strong signatures of nonclassical behavior. The initial quadratic behavior, also known as the Zeno regime, stems directly from a short-time expansion of the Schrödinger evolution, with the only hypotheses of normalizability of the wave function and finite energy fluctuations of the initial state. The familiar exponential decay sets in at intermediate times, and its derivation is always the consequence of assumptions of some sort, such as weak coupling or Markovianity. The long-time evolution is a consequence of the boundedness from below of the Hamiltonian, an indispensable condition from a physical perspective. Under this hypothesis, a straightforward application of the PaleyWiener theorem on Fourier transforms yields long-time power-law tails [6-8] (see, however, [9]).

The initial "Zeno" region [10] has been experimentally confirmed in a variety of physical systems, including trapped atoms [11-13], Bose-Einstein condensates [14,15], cavity quantum electrodynamics [16,17], Rydberg atoms [18], and optical waveguide arrays [19]. On the other hand, to the best of our knowledge, a single experimental observation of the power-law decay was reported, with the acquisition of the temporal decay of the fluorescence signal of dissolved organic molecules [20], although without an underlying theoretical model that enables one to compute the power-law decay from first principles. In general, power-law decay tails are very elusive, since the preceding region of exponential decay usually depletes the initial state at a point that makes any subsequent observation extremely challenging.

Arrays of single-mode optical waveguides are a powerful platform to experimentally investigate diverse quantum dynamics. The optical modes represent distinct quantum states of the photon that can be coupled with high control by tuning the evanescent-field-mediated interaction between waveguides. The time evolution of the Schrödinger equation is mapped onto the longitudinal propagation in the waveguides, thus making it easy to investigate even fast dynamics [21]. In addition, photons are almost immune to decoherence. Exploiting such favorable features, several quantum phenomena that are difficult to observe in solid state systems have been successfully studied with photonic structures. These include Bloch oscillations [22,23], Anderson localization [24,25], and the Zeno decay regime mentioned above [19]. 
(a)

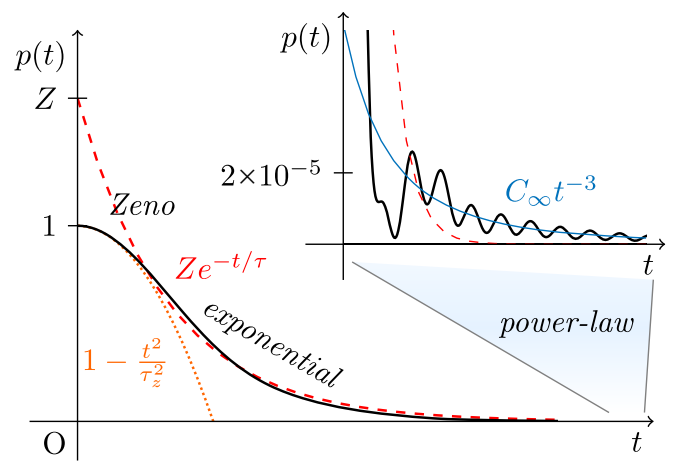

(b)

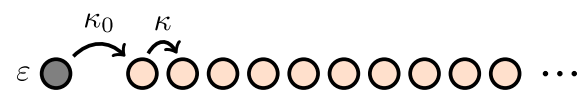

(c)

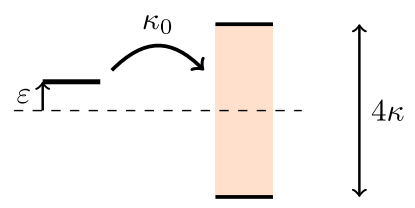

(d)

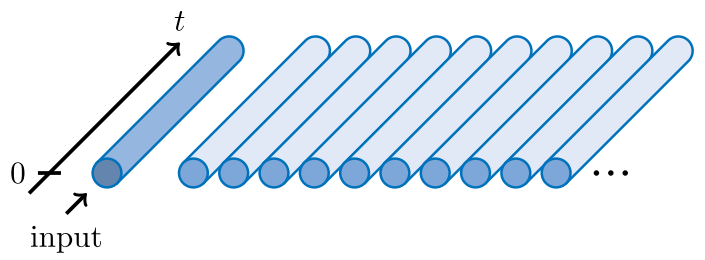

FIG. 1. (a) Typical decay of a quantum state coupled to a continuum, showing the peculiar features of the survival probability $p(t)$. The initial region is quadratic, with the curvature at $t=0$ characterized by the Zeno time $\tau_{Z}$. At intermediate times, a familiar exponential decay sets in, with a lifetime $\tau=O\left(\lambda^{-2}\right)$, where $\lambda$ is the coupling constant, and a "wave-function renormalization" $Z=1+O\left(\lambda^{2}\right)$, which represents the value of its extrapolation back to $t=0$. At very large times, when the survival probability is reduced to $O\left(\lambda^{10}\right)$, a power-law regime is observed, with superimposed oscillations. In particular, we refer to the system represented in (b): An optical mode with detuning $\varepsilon$ is coupled, by a coupling constant $\kappa_{0}$, to a chain of optical modes with relative coupling $\kappa$. (c) Level scheme of such a system: The states of the chain make up a continuum with bandwidth $4 \kappa$ (as in a tight-binding one-dimensional lattice). (d) Experimentally, we study this system with an array of singlemode optical waveguides.

Engineered waveguide arrays, excited with identical photon pairs, have also allowed the experimental study of multiparticle quantum decay processes [26].

In this work, we use optical waveguide arrays, fabricated by femtosecond laser micromachining technology [22, 26,27], to implement quantum systems where a discrete state is made unstable by its coupling to a continuum [28]. Different structures are fabricated, optimizing the parameters in such a way that different dynamical decay regimes can be detected and scrutinized, when coherent laser light is injected at the input. To probe the system evolution, we acquire with a high dynamic range the light scattered from the array. In this way, we are able to observe, within a single experimental platform, the quadratic Zeno region, the transition to the exponential regime, the wave-function renormalization, and the power-law decay at long evolution times.

The physical system investigated consists of a semiinfinite linear array of single-mode optical waveguides, which can be excited by light with fixed polarization. The transverse optical modes correspond to localized quantum states $|n\rangle$, with $n \geq 0$ indexing the different waveguides. Neighboring modes are coupled by evanescent-field interaction: The first one is coupled to the second one by a coefficient $\kappa_{0}$, while all others are coupled by a coefficient $\kappa$ [Fig. 1(b)]. The first waveguide is also characterized by a propagation-constant detuning $\varepsilon$, which, from the quantum evolution point of view, corresponds to the energy detuning of the site; all other waveguides are identical. As shown in Fig. 1(c), such a system actually consists in a discrete state (the first site), coupled to a continuum band of width $4 \kappa$ [29].

The dynamics of this quantum system is generated by the Hamiltonian

$$
H=H_{0}+H_{1}+H_{\text {int }},
$$

with

$$
\begin{aligned}
H_{0}= & \varepsilon|0\rangle\langle 0|, \\
H_{1}= & \kappa \sum_{n \geq 1}(|n\rangle\langle n+1|+| n+1\rangle\langle n|) \\
& +q \sum_{n \geq 1}(|n\rangle\langle n+2|+| n+2\rangle\langle n|), \\
H_{\text {int }}= & \kappa_{0}(|0\rangle\langle 1|+| 1\rangle\langle 0|)+q_{0}(|0\rangle\langle 2|+| 2\rangle\langle 0|) .
\end{aligned}
$$

Note that we have also included a next-nearest-neighbor hopping term, characterized by a coupling coefficient $q\left(q_{0}\right.$ for the first waveguide). This additional interaction is unavoidable in our experimental setting, and its effects are typically small [30] but can become quantitatively relevant in the high-depletion (long-time) regimes; we will assume for simplicity $q_{0}=q$ in the numerical simulations.

We consider the system initialized at $t=0$ in the first site of the array, $\left|\psi_{0}\right\rangle=|0\rangle$. The quantity typically chosen to investigate the temporal behavior of the system is the survival probability, defined (with $\hbar=1$ ) as

$$
p(t)=|a(t)|^{2}, \quad a(t)=\left\langle\psi_{0} \mid \psi(t)\right\rangle=\left\langle\psi_{0}\left|e^{-i t H}\right| \psi_{0}\right\rangle .
$$

The initial state is unstable, i.e., $p(t) \rightarrow 0$ as $t \rightarrow \infty$, if $\lambda^{2}<1-|\varepsilon| / 2 \kappa$ [31], being $\lambda=\kappa_{0} / \kappa$. The survival probability amplitude is the sum of two terms [31]:

$$
a(t)=\mathcal{Z} e^{-i E_{P} t}+a_{\text {cut }}(t)
$$


where $E_{P}$ is the pole of the propagator $G(z)=(z-H)^{-1}$ in the second Riemann sheet [28], whose imaginary component yields the decay rate. The exponential law is normally dominant at intermediate decay times. $\mathcal{Z}$ is called the wave-function renormalization and can be determined by extrapolating the exponential probability back to $t=0$ :

$$
Z=|\mathcal{Z}|^{2}=1+\frac{\lambda^{2}}{1-\lambda^{2}} \frac{1-\frac{3}{4} \lambda^{2}-\left(\frac{\varepsilon}{2 \kappa}\right)^{2}}{1-\lambda^{2}-\left(\frac{\varepsilon}{2 \kappa}\right)^{2}} .
$$

It is possible to check that, whenever the imaginary part of $E_{P}$ is nonvanishing, the value of $Z$ provided by the above equation is strictly larger than one. The term $a_{\text {cut }}(t)$ accounts for all deviations from the exponential and dominates at short and long times. In general, interference between the pole and cut terms also generates oscillations in the survival probability.

The survival probability at short times can be extracted by a Taylor expansion of the evolution operator $e^{-i t H}$, resulting in

$$
p(t)=1-\left(\frac{t}{\tau_{Z}}\right)^{2}+O\left(t^{4}\right) .
$$

The survival probability is thus quadratic at very short times, with curvature determined by the Zeno time

$$
\tau_{Z}=\left(\left\langle\psi_{0}\left|H^{2}\right| \psi_{0}\right\rangle-\left\langle\psi_{0}|H| \psi_{0}\right\rangle^{2}\right)^{-1 / 2}=\frac{1}{\kappa_{0}} .
$$

At long times, the contribution of $a_{\text {cut }}(t)$ becomes dominant over the exponential term, accounting for the power-law behavior. In our case, the survival probability at long times reads

$p(t) \simeq\left|a_{\mathrm{cut}}(t)\right|^{2}=\left(\frac{C(t)}{t}\right)^{3}\{1+\alpha(t) \cos [4 \kappa t+\varphi(t)]\}$,

where $C(t), \alpha(t)$, and $\varphi(t)$ become constant at sufficiently long times. This result is to be expected from first principles [6-8]. An exponential behavior at all times would imply a Lorentzian energy density distribution, with support on all (positive and negative) energies, i.e., an unbounded Hamiltonian. Instead, if the spectrum is bounded from below, with a finite ground-state energy, the Paley-Wiener theorem states that the function $[\ln p(t)] /\left(1+t^{2}\right)$ is integrable, and thus $p(t)$ must be slower than exponential at long enough times. In general, the power law appearing in Eq. (10) is related to the structure of the coupling and the behavior of the density of states at energies close to the edge(s) of the continuum. The oscillatory behavior, with angular frequency $4 \kappa$, is due to the interference between the contributions from the two band edges. Finally, notice that the exact expression of the Zeno time (9) is left unchanged by the introduction of next-to-nearest-neighbor couplings, as well as the form of the long-time survival probability (10), provided $q$ is real and $|q / \kappa|<1 / 4$.

In our experiments, waveguides were fabricated in a fused silica substrate by femtosecond laser direct inscription [32]. We used the second harmonic (520 nm wavelength) of an ytterbium femtosecond laser (HighQ femtoREGEN), producing $\simeq 400 \mathrm{fs}$ duration pulses at $20 \mathrm{kHz}$ repetition rate. In our experiments, laser pulses with $350 \mathrm{~nJ}$ energy were focused, by means of a $0.45 \mathrm{NA}$ microscope objective, $170 \mu \mathrm{m}$ below the glass surface, and the substrate was translated with respect to the laser beam at a constant speed comprised between 20 and $34 \mathrm{~mm} / \mathrm{s}$. Waveguides fabricated with these irradiation parameters yield single-mode behavior for the $633 \mathrm{~nm}$ wavelength and propagation losses of $0.6 \mathrm{~dB} / \mathrm{cm}$.

To investigate the light propagation in waveguide arrays, we injected horizontally polarized light from a $\mathrm{He}: \mathrm{Ne}$ laser source in the first waveguide (which corresponds to initializing the system in state $|0\rangle$ ) and imaged the structure from above, acquiring the scattered light at each point. The scattered signal is indeed locally proportional to the intensity of the propagating light.

As mentioned in the preceding analysis, we are mainly interested in retrieving the population of the first waveguide. This is not easy, because such a quantity spans a few orders of magnitude: At its entrance (i.e., at $t=0$ ), the first waveguide (namely, state $|0\rangle$ ) is fully populated, but at later times, when the interesting power-law dynamics sets in, it may be heavily depleted. To perform the measurement, we developed a microscope assembly operating as a highdynamic-range image scanner [33]. The assembly is moved along the propagation coordinate by a computer-controlled motorized stage, with synchronized image acquisition by a $\mathrm{CCD}$. To enhance the dynamic range of the measurement, pictures taken at different exposure times are combined and analyzed together. The experimental survival probability $p(t)$ is retrieved as the ratio between the optical power scattered from the first waveguide and the global scattered power at each $t$. In this way, propagation losses of the waveguides, which are uniform in the array, are also normalized out and do not affect our results.

The coupling coefficients $\kappa, \kappa_{0}$, and $q$ depend on the relative distance between the waveguides, while the detuning $\varepsilon$ can be controlled by varying the writing speed [32]. These quantities have been calibrated in independent experiments, where we fabricated several couples of identical parallel waveguides at different relative distances and other couples of parallel waveguides that differ in writing speed. By observing the periodicity of the bouncing of coherent light between the coupled waveguides [34], it is possible to measure the coupling coefficients and the propagation-constant detuning, thus retrieving their dependence on the inscription parameters.

We realized arrays characterized by different geometrical parameters, each containing 40 waveguides. To avoid boundary effects, we always chose coupling conditions in which 
TABLE I. Relevant physical parameters of the three waveguide arrays $(A, B$, and $C) . d_{0}$ is the distance between the first and the second waveguide, and $d$ the distance between all other neighboring waveguides. $v_{0}$ is the writing speed of the first waveguide, and $v$ the writing speed of all the other ones. $\kappa_{0}, \kappa, \varepsilon$, and $q$ have the same meaning as in Eq. (5); the reported values for each array are the nominal ones, estimated on the basis of the preliminary calibration experiments. Errors correspond to standard deviations and are due to tolerances in the waveguide inscription process; they are also estimated by means of the calibration experiments. Where not written explicitly, uncertainty is indicated by the number of significant digits used.

\begin{tabular}{ccccccccc}
\hline \hline & $d_{0}$ & $d$ & $v_{0}$ & $v$ & $\kappa_{0}$ & $\kappa$ & $q$ & $q$ \\
\hline$A$ & $12.0 \mu \mathrm{m}$ & $17.0 \mu \mathrm{m}$ & $31.30 \mathrm{~mm} / \mathrm{s}$ & $30.00 \mathrm{~mm} / \mathrm{s}$ & $0.045 \pm 0.001 \mathrm{~mm}^{-1}$ & $0.119 \pm 0.004 \mathrm{~mm}^{-1}$ & $-0.08 \pm 0.09 \mathrm{~mm}^{-1}$ & $0.005 \mathrm{~mm}^{-1}$ \\
$B$ & $15.5 \mu \mathrm{m}$ & $15.0 \mu \mathrm{m}$ & $30.00 \mathrm{~mm} / \mathrm{s}$ & $31.30 \mathrm{~mm} / \mathrm{s}$ & $0.118 \pm 0.004 \mathrm{~mm}^{-1}$ & $0.132 \pm 0.004 \mathrm{~mm}^{-1}$ & $0.10 \pm 0.09 \mathrm{~mm}^{-1}$ & $0.01 \mathrm{~mm}^{-1}$ \\
$C$ & $14.0 \mu \mathrm{m}$ & $15.0 \mu \mathrm{m}$ & $30.00 \mathrm{~mm} / \mathrm{s}$ & $30.00 \mathrm{~mm} / \mathrm{s}$ & $0.183 \pm 0.006 \mathrm{~mm}^{-1}$ & $0.158 \pm 0.005 \mathrm{~mm}^{-1}$ & $0.0 \mathrm{~mm}^{-1}$ & $0.01 \mathrm{~mm}^{-1}$ \\
\hline \hline
\end{tabular}

light does not reach the last waveguide of the array within the propagation length $(<9 \mathrm{~cm}$, the size of our glass samples) [35]. In such conditions, the system dynamics is well explained by the semi-infinite model discussed above. Table I displays the relevant physical parameters of three structures, which have been tailored in order to observe different dynamical regimes.

The transition between the initial Zeno region and the exponential decay is evident for systems in which state $|0\rangle$ is weakly coupled to the continuum. In Fig. 2, we report the experimental decay of a system designed with $\lambda \simeq 0.37$, whose parameters are listed in line $A$ in Table I. Figure 2(a) shows, in linear scale, the full evolution analyzed in the experiment. An initial quadratic region is manifest at early
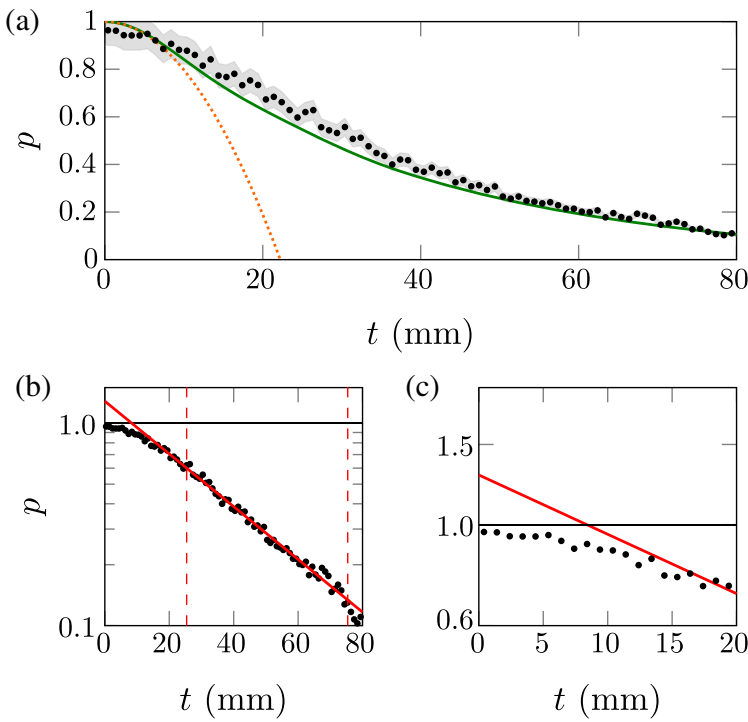

FIG. 2. Experimental survival probability in a weakly coupled system (array $A$ in Table I). Experimental points (black dots) are reported in linear (a) and semilogarithmic plots (b),(c). Experimental errors are shown in the linear plot as a gray area around the points. The green continuous line in (a) is the theoretical prediction, obtained by solving Eq. (5) with the nominal data $\left(\kappa_{0}, \kappa, \varepsilon, q\right)$ of array $A$. The orange dotted line in (a) is the parabolic trend $1-\left(t / \tau_{z}\right)^{2}$ with $\tau_{z}=1 / \kappa_{0}$. The red continuous line in (b) and (c) is a linear fit performed on the semilogarithmic plot, considering the data between the two dashed red lines in (b). times. The subsequent exponential behavior is plainly revealed in Fig. 2(b), where the same data are plotted in a semilogarithmic graph: A linear fit, corresponding to an exponential decay in the linear scale, is also plotted. By enlarging the propagation region below $20 \mathrm{~mm}$ [Fig. 2(c)], one can see that the intercept of this straight line, corresponding to the wave-function renormalization parameter $Z$ in Eq. (7), falls above 1. Although the fitted value $Z \simeq 1.23$ is slightly larger than expected, the experimental outcomes confirm the theoretical prediction $Z>1$ for the analyzed dynamics, independent of the specific values of the parameters.

The large-time behavior predicted in Eq. (10), consisting in a $t^{-3}$ power-law tail with superimposed oscillations, can be better appreciated in systems with stronger couplings. Figure 3 shows the measured decay for two systems, corresponding to cases $B$ and $C$ in Table I and featuring $\lambda \simeq 0.89$ and $\lambda \simeq 1.16$, respectively. In case $C$, the energy detuning of the system is zero (waveguides are written with the same propagation constant) and oscillations are more pronounced, while in case $B$, where the detuning is relevant $(\varepsilon \simeq \kappa)$, oscillations are almost suppressed.

In case $B$, the experimentally observed decay follows with good approximation the theoretical (solid green) line, which, at $t \simeq 40 \mathrm{~mm}$, relaxes towards the asymptotic power law $p(t) \simeq\left(C_{\infty} / t\right)^{3}$ [see Eq. (10)], with $C_{\infty}=9.48 \mathrm{~mm}$. Thus, the dynamics of case $B$ features a pure power-law behavior at times which are long but still within reach of the experiment. It must be noted that the choice of the parameters has allowed the observation of the onset of such a regime when the state was not heavily depleted yet $\left[p(t=40 \mathrm{~mm}) \sim 10^{-2}\right]$. Even for case $C$, theoretical simulations show that the power law takes place at sufficiently long times, but here such times are far beyond the experimental reach by an order of magnitude. Therefore, the behavior observed in Fig. 3(b), although subexponential, cannot be described by a single power law. The most interesting feature of the time evolution in case $C$ is the presence of oscillations with period $\pi /(2 \kappa)$ [see Eq. (10)], which are due to the coupling with a bounded continuum, with bandwidth $4 \kappa$. Such oscillations are entirely due to the cut contributions to the survival amplitude and cannot be described by any Markovian approximation. 

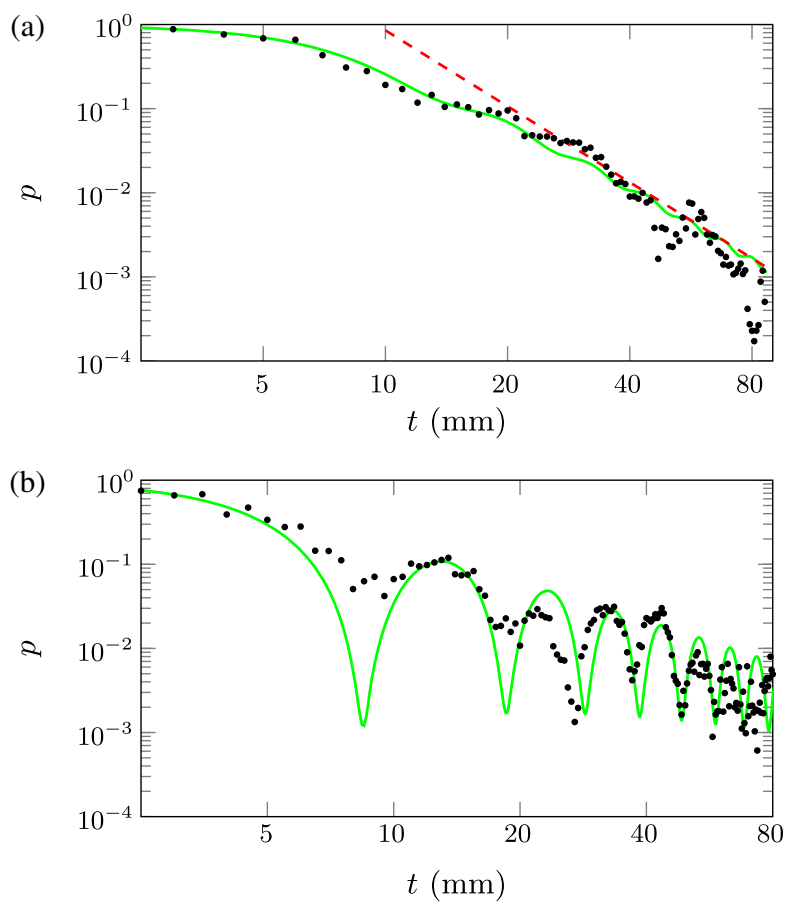

FIG. 3. Experimental survival probability in systems with stronger coupling, i.e., array $B[(\mathrm{a})]$ and array $C[(\mathrm{~b})]$ in Table I. Experimental points (black dots) are reported in logarithmic plots; experimental errors are comparable to the marker size. The (green) solid lines represent data fitting, obtained by solving Eq. (5) using (a) $\kappa_{0}=0.119 \mathrm{~mm}^{-1}, \kappa=0.132 \mathrm{~mm}^{-1}, \varepsilon=0.12 \mathrm{~mm}^{-1}$, and $q=0.01 \mathrm{~mm}^{-1}$ and (b) $\kappa_{0}=0.205 \mathrm{~mm}^{-1}, \kappa=0.160 \mathrm{~mm}^{-1}$, $\varepsilon=0 \mathrm{~mm}^{-1}$, and $q=0.01 \mathrm{~mm}^{-1}$; the fitting parameters are close to the nominal ones reported in Table I. The (red) dashed line in (a) represents the polynomial trend $p(t) \simeq\left(C_{\infty} / t\right)^{3}$.

We have reported the experimental observation of different decay regimes, typical of genuinely quantum dynamics, using photons propagating in waveguide arrays. The femtosecond laser writing technology allowed us to define with high control the relevant physical properties of the system. We adopted a multiexposure acquisition technique to extend the available dynamic range beyond the 8-bit limit of the camera, allowing us to compare intensity levels which differ by a factor larger than $10^{4}$. In this way, we have been able to characterize power-law decay tails, which are generally very elusive to experimental observation. We believe that these results open novel perspectives in the study of quantum decay dynamics, as well as in the investigation of the interaction between a system and its environment, including noise-enhanced transport phenomena or non-Markovian processes.

R. O. acknowledges financial support by the European Research Council (ERC) Advanced Grant CAPABLE (Grant Agreement No. 742745). P. F. and S. P. are partially supported by Instituto Nazionale di Fisica Nucleare (INFN) through the project "QUANTUM." F. V. P. is supported by
INFN through the project "PICS." P.F. is partially supported by the Italian National Group of Mathematical Physics (GNFM-INdAM).

* Corresponding author. roberto.osellame@polimi.it

[1] E. B. Norman, S. B. Gazes, S. G. Crane, and D. A. Bennett, Tests of the Exponential Decay Law at Short and Long Times, Phys. Rev. Lett. 60, 2246 (1988).

[2] D. Novković, L. Nadderd, A. Kandić, I. Vukanac, M. Durašević, and D. Jordanov, Testing the exponential decay law of gold 198Au, Nucl. Instrum. Methods Phys. Res., Sect. A 566, 477 (2006).

[3] H. Nakazato, M. Namiki, and S. Pascazio, Temporal behavior of quantum mechanical systems, Int. J. Mod. Phys. B 10, 247 (1996).

[4] P. Facchi and S. Pascazio, Quantum Zeno and inverse quantum Zeno effects, in Progress in Optics, edited by E. Wolf (Elsevier, Amsterdam, 2001), Vol. 42, p. 147.

[5] P. Facchi and S. Pascazio, Quantum Zeno dynamics: Mathematical and physical aspects, J. Phys. A 41, 493001 (2008).

[6] L. A. Khalfin, On the theory of the decay of a quasistationary state, Sov. Phys. Dokl. 2, 340 (1957).

[7] L. A. Khalfin, Contribution to the decay theory of a quasistationary state, Sov. Phys. JETP 6, 1053 (1958).

[8] P. Exner, Open Quantum Systems and Feynman Integrals (Reidel, Dordrecht, 1985).

[9] D. Burgarth and P. Facchi, Positive Hamiltonians can give purely exponential decay, Phys. Rev. A 96, 010103 (2017).

[10] B. Misra and E. C. G. Sudarshan, The Zeno's paradox in quantum theory, J. Math. Phys. (N.Y.) 18, 756 (1977).

[11] S. R. Wilkinson, C. F. Bharucha, M. C. Fischer, K. W. Madison, P. R. Morrow, Q. Niu, B. Sundaramand, and M. G. Raizen, Experimental evidence for non-exponential decay in quantum tunnelling, Nature (London) 387, 575 (1997).

[12] M. C. Fischer, B. Gutiérrez-Medina, and M. G. Raizen, Observation of the Quantum Zeno and Anti-Zeno Effects in an Unstable System, Phys. Rev. Lett. 87, 040402 (2001).

[13] Y. Lin, J. P. Gaebler, F. Reiter, T. R. Tan, R. Bowler, Y. Wan, A. Keith, E. Knill, S. Glancy, K. Coakley, A. S. Sorensen, D. Leibfried, and D. J. Wineland, Preparation of Entangled States through Hilbert Space Engineering, Phys. Rev. Lett. 117, 140502 (2016).

[14] E. W. Streed, J. Mun, M. Boyd, G. K. Campbell, P. Medley, W. Ketterle, and D. E. Pritchard, Continuous and Pulsed Quantum Zeno Effect, Phys. Rev. Lett. 97, 260402 (2006).

[15] F. Schäfer, I. Herrera, S. Cherukattil, C. Lovecchio, F. S. Cataliotti, F. Caruso, and A. Smerzi, Experimental realization of quantum zeno dynamics, Nat. Commun. 5, 3194 (2014).

[16] J.-M. Raimond, C. Sayrin, S. Gleyzes, I. Dotsenko, M. Brune, S. Haroche, P. Facchi, and S. Pascazio, Phase Space Tweezers for Tailoring Cavity Fields by Quantum Zeno Dynamics, Phys. Rev. Lett. 105, 213601 (2010).

[17] G. Barontini, L. Hohmann, F. Haas, J. Estève, and J. Reichel, Deterministic generation of multiparticle 
entanglement by quantum Zeno dynamics, Science $\mathbf{3 4 9}$, 1317 (2015).

[18] A. Signoles, A. Facon, D. Grosso, I. Dotsenko, S. Haroche, J.-M. Raimond, M. Brune, and S. Gleyzes, Confined quantum Zeno dynamics of a watched atomic arrow, Nat. Phys. 10, 715 (2014).

[19] P. Biagioni, G. Della Valle, M. Ornigotti, M. Finazzi, L. Duo, P. Laporta, and S. Longhi, Experimental demonstration of the optical Zeno effect by scanning tunneling optical microscopy, Opt. Express 16, 3762 (2008).

[20] C. Rothe, S. I. Hintschich, and A. P. Monkman, Violation of the Exponential-Decay Law at Long Times, Phys. Rev. Lett. 96, 163601 (2006).

[21] S. Longhi, Quantum optical analogies using photonic structures, Laser Photonics Rev. 3, 243 (2009).

[22] N. Chiodo, G. Della Valle, R. Osellame, S. Longhi, G. Cerullo, R. Ramponi, P. Laporta, and U. Morgner, Imaging of Bloch oscillations in erbium-doped curved waveguide arrays, Opt. Lett. 31, 1651 (2006).

[23] H. Trompeter, W. Krolikowski, D. N. Neshev, A. S. Desyatnikov, A. A. Sukhorukov, Yu. S. Kivshar, T. Pertsch, U. Peschel, and F. Lederer, Bloch Oscillations and Zener Tunneling in Two-Dimensional Photonic Lattices, Phys. Rev. Lett. 96, 053903 (2006).

[24] L. Martin, G. Di Giuseppe, A. Perez-Leija, R. Keil, F. Dreisow, M. Heinrich, S. Nolte, A. Szameit, A. F. Abouraddy, D. N. Christodoulides, and B. E. A. Saleh, Anderson localization in optical waveguide arrays with off-diagonal coupling disorder, Opt. Express 19, 13636 (2011).

[25] Y. Lahini, A. Avidan, F. Pozzi, M. Sorel, R. Morandotti, D. N. Christodoulides, and Y. Silberberg, Anderson Localization and Nonlinearity in One-Dimensional Disordered Photonic Lattices, Phys. Rev. Lett. 100, 013906 (2008).
[26] A. Crespi, L. Sansoni, G. Della Valle, A. Ciamei, R. Ramponi, F. Sciarrino, P. Mataloni, S. Longhi, and R. Osellame, Particle Statistics Affects Quantum Decay and Fano Interference, Phys. Rev. Lett. 114, 090201 (2015).

[27] A. Szameit and S. Nolte, Discrete optics in femtosecondlaser-written photonic structures, J. Phys. B 43, 163001 (2010).

[28] C. Cohen-Tannoudji, J. Dupont-Roc, and G. Grynberg, Atom-Photon Interactions: Basic Processes and Applications (Wiley, New York, 1998).

[29] This is true also in the case in which next-to-nearestneighbor waveguides are coupled, with coupling constant $q$ [see Eq. (1)], provided that $|q / \kappa|<1 / 4$.

[30] R. Keil, B. Pressl, R. Heilmann, M. Gräfe, G. Weihs, and A. Szameit, Direct measurement of second-order coupling in a waveguide lattice, Appl. Phys. Lett. 107, 241104 (2015).

[31] See Supplemental Material at http://link.aps.org/ supplemental/10.1103/PhysRevLett.122.130401 for further details in Sec. I.

[32] A. Crespi, S. Longhi, and R. Osellame, Photonic Realization of the Quantum Rabi Model, Phys. Rev. Lett. 108, 163601 (2012).

[33] See Supplemental Material at http://link.aps.org/ supplemental/10.1103/PhysRevLett.122.130401 for further details on the characterization method and on the experimental apparatus in Sec. II.

[34] A. Yariv, Coupled-mode theory for guided-wave optics, IEEE J. Quantum Electron. 9, 919 (1973).

[35] See, as a significant example, Fig. 3 in Supplemental Material at http://link.aps.org/supplemental/10.1103/ PhysRevLett.122.130401. 